\title{
Two all-optical logic gates in a single photonic interferometer
}

\author{
Antônio Araújo ${ }^{\mathrm{a}}$, Antônio Oliveira ${ }^{\mathrm{a}}$, Francisco Martins ${ }^{\mathrm{a}}$, Amarílio Coelho ${ }^{\mathrm{b}}$, Wilton Fraga ${ }^{\mathrm{b}}$, \\ José Nascimento ${ }^{\text {a,* }}$ \\ a Federal University of Ceará, Campus Mucambinho, Sobral, Ceará, Brazil \\ ${ }^{\mathrm{b}}$ Federal Institute of Education of Ceará, Campus Sidal, Sobral, Ceará, Brazil
}

\section{A R T I C L E I N F O}

\section{Article history:}

Received 9 April 2015

Accepted 4 July 2015

Available online 16 July 2015

Keywords:

Mach-Zehnder interferometer (MZI)

Photonic crystal fiber

Nonlinear directional coupler (NLDC)

Pulse amplitude modulation (PAM)

All-optical logic gate

\begin{abstract}
A B S T R A C T
In this paper is presented the all-optical AND and OR gates with high contrast ratio in a single interferometric configuration, i.e., when two logic signals are modulated in the input of the interferometer, so we have the OR gate in the first output and the AND gate in the second output. These logic gates were obtained by numerical investigation of the Mach-Zehnder interferometer constituted of dual-core nonlinear photonic crystal fiber operating with ultrashort fundamental solitons of 100 fs. To represent the logic information, pulse amplitude modulation by amplitude shift-keying was used.
\end{abstract}

(c) 2015 Elsevier B.V. All rights reserved.

\section{Introduction}

The photonic crystal fibers (PCF) constitute a new class of optical fibers. It is formed by materials with low and high refractive index. The material which composes the core of PCF is non-doped silica. The region of low refractive index is composed of a periodic array of air holes which extends throughout its entire length. Because of its features, PCFs have found many important applications such as dispersion control [1], endless single-mode operation [2] and super-continuum generation [3].

It has been shown theoretically and experimentally that the dual-core optical fiber employs two central coupled regions which can be used as a directional optical coupler [4,5]. The nonlinear directional coupler (NLDC) constituted of dual-core optical fibers offers possibilities for switching, routing and modulation of optical signals over the nonlinear interaction in the coupling region between the wave guides [6,7]. Therefore, some of these properties are expected and can be improved in dual-core photonic crystal fiber. Its structure is shown in Fig. 1 . The distance $C$ separates the two cores, the hole-to-hole space is $\Lambda$ and the diameter of air holes is $d$.

A full model for ultrashort pulse propagation in dual-core PCF to explain the experimental results has been presented in [8]. In [9] was made the correction in the term referring to the coupling coefficient dispersion and it was found that this term being much

\footnotetext{
* Corresponding author.

E-mail address: claudio.nasce@gmail.com (J. Nascimento).

URL: http://www.ppgeec.ufc (J. Nascimento).
}

shorter than one meter is sufficient to cause significant distortion for ultrashort input pulses. After these two papers, investigations have been made to some applications in all-optical logic gates. In [10] has been proposed a logic gate OR using amplitude shift keying (ASK) with length propagation $1.8 \mathrm{~cm}$. To emphasize the importance of this result, a similar result was previously obtained in dual-core optical fiber with propagation of $64.3 \mathrm{~m}$ in [11]. In [12] the AND and OR gates were obtained in the numerical study of triple-core PCF with ASK modulation where one of the three cores receive a pulse to activate the logic gates. In addition, investigations using on-off keying modulation were performed [13].

In this paper, we have investigated numerically the behavior of the Mach-Zehnder interferometer composed of two NLDC (Fig. 2) to obtain logic gates. All-optical AND and OR gates were found without a pulse to activate the logic gates to ASK (passive logic gates to ASK). The contrast ratio to AND and OR gates are high, showing the high performance to both logic gates. Furthermore, the OR and AND gates are found in the same configuration of MZI, i.e., we have two logic gates in the same device. This is a topic of attractive research because two logic gates in the same interferometer are useful to saving-space in complex circuit designs and ultrashort pulses are necessary to increase the performance of processing information. Furthermore, the Mach-Zehnder interferometer with dual-core photonic crystal fiber has been implemented experimentally [14].

This work is organized as follows: in Section 2 we show the theoretical modeling based on the pair of coupled mode equations; in Section 3 is presented the PAM-ASK for hyperbolic secant pulses; in Section 4 we present the details of the numerical 


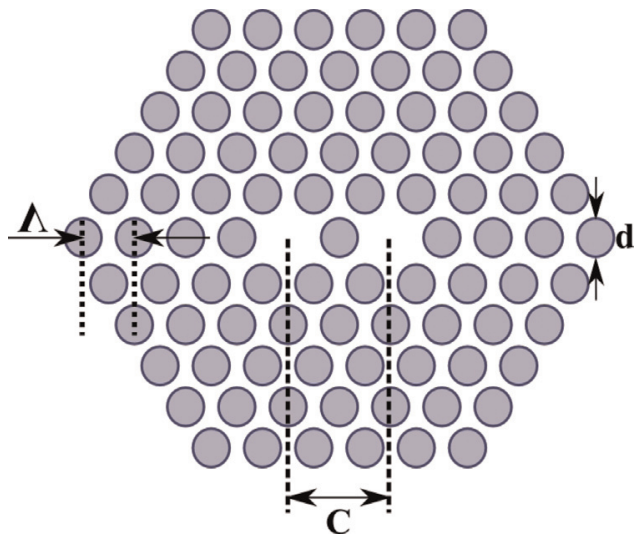

Fig. 1. Cross-section schematic of the dual-core photonic crystal fiber.

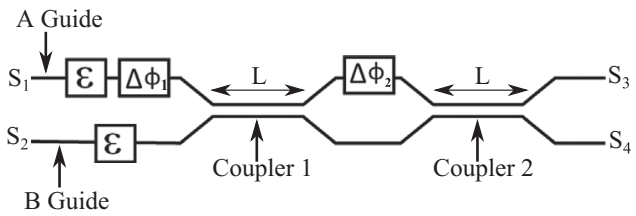

Fig. 2. Schematic of the proposed system. Mach-Zehnder Interferometer constituted of photonic crystal fibers.

method applied in the solution of the pair of coupled mode equations; in Section 5 the study of energy transmission in the Mach-Zehnder interferometer is analyzed to verify which parameters can be used to implement amplitude shift-keying in the output; in Section 6 we present a discussion about the found results; finally, in Section 7 we present the conclusion.

\section{Theoretical model}

In this study, to form the Mach-Zehnder interferometer it is necessary to cascade two nonlinear couplers (see Fig. 2). The first coupler divides the input signals into two parts that interact with each other and soon after the phase shift is applied. Then, in the second coupler, the two pulses interfere with each other and they are routed to the output. The ports $S_{1}$ and $S_{2}$ are the inputs and the ports $S_{3}$ and $S_{4}$ are the outputs. There are two modulators $(\varepsilon)$ performing pulse amplitude modulation with amplitude shiftkeying (PAM-ASK) and two phase controls $\left(\Delta \Phi_{1}\right.$ and $\left.\Delta \Phi_{2}\right)$.

The nonlinear coupled mode equation comes from the Nonlinear Schrödinger Equation (NLSE). It rules the behavior of electromagnetic fields in a coupler with a high nonlinearity and high order dispersion effects. Then, for an envelope propagating in a nonlinear directional coupler (NLDC) made of PCF and operating without loss (due to the propagation length), we have

$$
\begin{array}{r}
i \frac{\partial a_{1}}{\partial z}-\frac{\beta_{2}}{2} \frac{\partial^{2} a_{1}}{\partial t^{2}}-i \frac{\beta_{3}}{6} \frac{\partial^{3} a_{1}}{\partial t^{3}}+\frac{\beta_{4}}{24} \frac{\partial^{4} a_{1}}{\partial t^{4}}+\gamma\left(\left|a_{1}\right|^{2}+\eta\left|a_{2}\right|^{2}\right) \\
+i \frac{\gamma}{\omega} \frac{\partial\left(a_{1}\left|a_{1}\right|^{2}\right)}{\partial t}-\gamma a_{1} T_{R} \frac{\partial\left|a_{1}\right|^{2}}{\partial t}+\kappa_{0} a_{2}+i \kappa_{1} \frac{\partial a_{2}}{\partial t}=0 \\
i \frac{\partial a_{2}}{\partial z}-\frac{\beta_{2}}{2} \frac{\partial^{2} a_{2}}{\partial t^{2}}-i \frac{\beta_{3}}{6} \frac{\partial^{3} a_{2}}{\partial t^{3}}+\frac{\beta_{4}}{24} \frac{\partial^{4} a_{2}}{\partial t^{4}}+\gamma\left(\left|a_{2}\right|^{2}+\eta\left|a_{1}\right|^{2}\right) \\
+i \frac{\gamma}{\omega} \frac{\partial\left(a_{2}\left|a_{2}\right|^{2}\right)}{\partial t}-\gamma a_{2} T_{R} \frac{\partial\left|a_{2}\right|^{2}}{\partial t}+\kappa_{0} a_{1}+i \kappa_{1} \frac{\partial a_{1}}{\partial t}=0,
\end{array}
$$

where $z$ is the distance along the fiber. $t$ is the time coordinate with reference to the transit time of the pulses. $a_{1}$ and $a_{2}$ are the amplitude envelopes of the pulses carried by the two cores, respectively. $\beta_{2}, \beta_{3}$ and $\beta_{4}$ are the group-velocity dispersion (GVD), third-order dispersion (TOD), and fourth-order dispersion, respectively. $\gamma$ is the nonlinear parameter that accounts for selfphase modulation (SPM). $\eta$ is a small ratio that measures the relative importance of cross-phase modulation (XPM) with regard to SPM. The time-varying term next to the SPM and XPM terms represents self-steepening (where $\omega$ is the angular optical frequency). $T_{R}$ is the Raman scattering coefficient. $\kappa_{0}$ is the coupling coefficient and $\kappa_{1}$ is the coupling coefficient dispersion given by $\kappa_{1}=d \kappa_{0} / d \omega$ (evaluated at the pulse carrier frequency). The coupling coefficient dispersion $\kappa_{1}$ is a measure of the wavelength dependence of the coupling coefficient. It is equivalent to the intermodal dispersion of the composite two-mode fiber structure $[9,15,16]$.

The photonic crystal fibers have a high nonlinearity. The following equation describes the effective nonlinear parameter

$\gamma=\frac{2 \pi \eta_{2}}{\lambda A_{\text {eff }}}$

where $A_{e f f}$ is the effective mode area and $\lambda$ is the wavelength. In this study, we have not considered saturation effects of the nonlinearity for intense fields $[17,18]$. According to Eq. (3), a decrease of effective area increases the nonlinearity. That makes the PCF very nonlinear compared to the conventional optic fiber. Due to the presence of materials with different features of nonlinearity, the following equation gives the effective area of PCF:

$A_{e f f}=\frac{\eta_{2}\left[\iint E(x, y) \cdot E^{*}(x, y) d x d y\right]^{2}}{\iint \bar{\eta}_{2}(x, y)\left[E(x, y) \cdot E^{*}(x, y)\right]^{2} d x d y}$,

where $E(x, y)$ is the transverse electric field and $\eta_{2}(x, y)$ is the nonlinear coefficient of the material. Effective nonlinearities of the order of $640 \mathrm{~W}^{-1} \mathrm{~km}^{-1}$ have been reported in a solid core PCF [19].

The interaction between the propagating fields in a NLDC occurs by transmission and coupling of pulses that propagate through it. For this, it is necessary that the distance between the two guides is in the order of decay of evanescent radiation [17]. The input power is critical in the coupling process between the guides. When a port of the coupler is excited with a high power, beyond the critical power, the light will not be transmitted to the other guide, following then, through the same guide. However, when we activate the guide with a low power, all the energy is transmitted to the other guide [18]. The length of the coupling $L_{C}$ is that in which a low power pulse propagating in one of the guides is totally switched to the other core [15]. This length is defined by $L_{C}=2 \pi / \kappa$ where $\kappa$ is the linear coupling between the two guides.

When the light incidence in a guide is low, the device behaves as a linear coupler, i.e. the optical ray propagates periodically between the guides that forms the coupler. However, the transmission features are destroyed to high incident power values due to the change in the refractive index. When the incident power is the same as critical power, the light will emerge equally in both NLDC's outputs [15]. That block on transmission happens for powers beyond the critical power:

$P_{C}=\frac{A_{e f f} \lambda}{\eta_{N L} L_{C}}$

where $\eta_{N L}$ is the nonlinear refractive index and $L_{C}$ is the necessary coupling length to the full energy transfer from a guide to the other. 


\section{Pulse amplitude modulation with amplitude shift keying}

The pulse amplitude modulation with amplitude shift keying (PAM-ASK) in this paper is able to transmit one symbol for each sent pulse. We had utilized $a(z, t)$ as the reference pulse in the hyperbolic secant with an amplitude $a_{0}[4,6,12]$. The modulated signals have the same form of the reference pulse, but the amplitudes $\left(a_{0}+\varepsilon\right)$ and $\left(a_{0}-\varepsilon\right)$ represent the bit 1 and bit 0 , respectively, where $\varepsilon$ is the coding parameter. Then, the pulses in ASK are defined as follows:

$a_{R E F}(0, t)=a_{0} \operatorname{sech}(t)$

$a_{\text {ZERO }}(0, t)=\left(a_{0}-\varepsilon\right) \operatorname{sech}(t)$,

$a_{\text {ONE }}(0, t)=\left(a_{0}+\varepsilon\right) \operatorname{sech}(t)$.

We have the form of the pulses before and after the modulators $\varepsilon$ (see Fig. 2). Then, for PAM-ASK, every pulse with an amplitude greater than the reference amplitude is considered as the logic level 1 and every pulse with an amplitude smaller than the reference amplitude is considered as the logic level 0 .

\section{Numerical method}

We investigated the performance of the device showed in Fig. 2. The system is composed of two NLDC-PCF and two PAMASK modulators. The parameter of the modulator is defined as $\varepsilon$
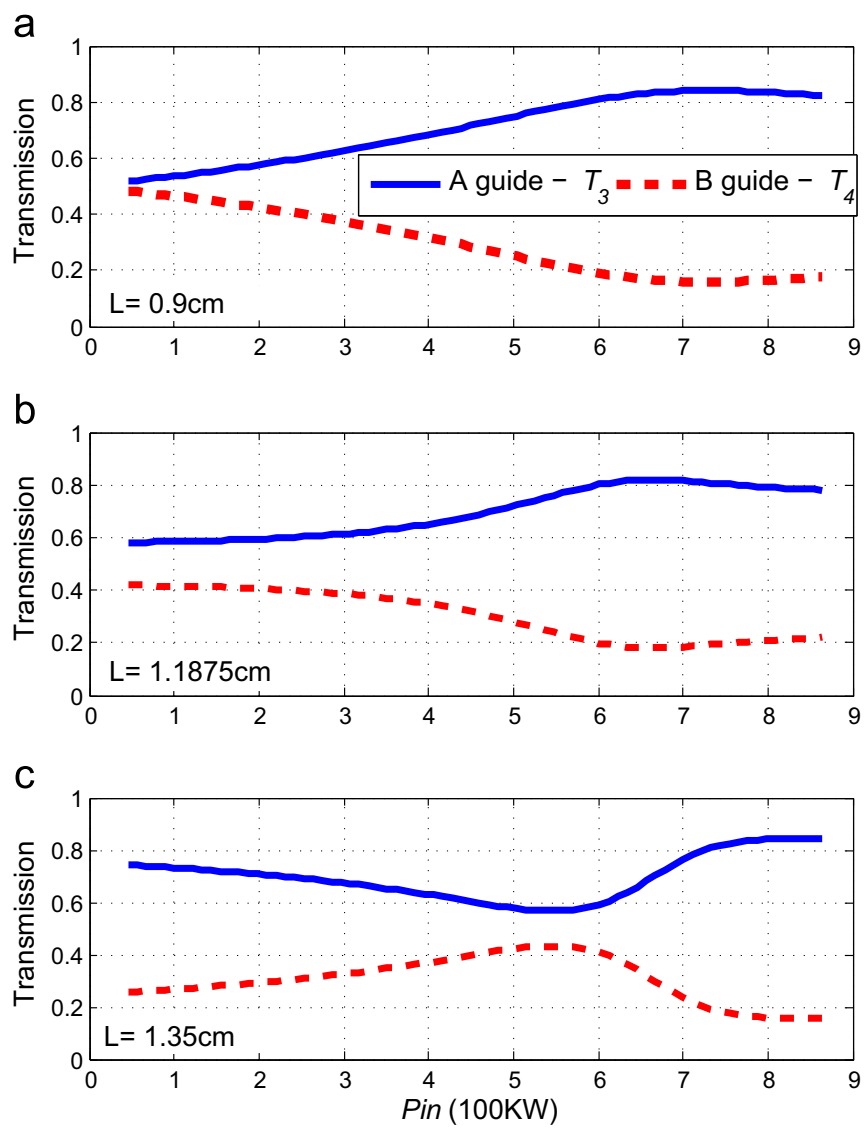

Fig. 3. The transmissions $T_{3}$ and $T_{4}$ as a function of the input power pulse $\left(P_{\text {in }}\right)$ Three numerical simulations are realized for $\Delta \Phi_{2}=0.4 \pi$ (a), $\Delta \Phi_{2}=0.6 \pi$ (b) and $\Delta \Phi_{2}=0.8 \pi$ (c). The parameters $\Delta \Phi_{1}=0$ and $L=1.1875 \mathrm{~cm}$ are fixed for all simulations. and represents the displacement of the amplitude for definition of its logic value. After passing through the PAM-ASK modulator, the pulse enters the NLDC-PCF as shown below:

$S_{k}=\left[a_{0}+(-1)^{1-b} \varepsilon\right] \operatorname{sech}(t)$,

where the index $k=1,2$ makes a reference to the ports 1 and 2 respectively, $a_{0}=\sqrt{P_{0}}$ is the amplitude, $P_{0}$ is the peak power of the pulse and $\varepsilon$ is the encoding parameter. When $b$ represents the logic value 1 , we will have $\left(a_{0}+\varepsilon\right) \operatorname{sech}(t)$. When $b$ is the logic value 0 , we will have $\left(a_{0}-\varepsilon\right) \operatorname{sech}(t)$. The amplitude of the modulated pulses is calculated from the temporal position of its respective maximal intensity.

According to Eqs. (1) and (2) the reference frame moves with a group velocity $\left(v_{g}\right)$, so the time is $t=t^{\prime}-z / v_{g}$ [9]. We used the fundamental soliton propagation, i.e. the first order soliton. So, the non-linearity length is the same as dispersion length $\left(L_{N L}=L_{D}\right)$. We applied full temporal width at half maximum (TFWHM) of the input pulse $\Delta t=100 \mathrm{fs}$. We neglected the fourth order dispersion and operated in negligible loss regime due to the small length of the fiber. We have analyzed two couplers of dual-core photonic crystal fibber, constituted of silica, with air-hole diameter of $d=2 \mu \mathrm{m}$, hole-to-hole distance $\Lambda=d / 0.9$, distance between the cores $C=2 \Lambda$, coupling length $L_{C}=1.8 \mathrm{~cm}$, wavelength close to the region $\lambda=1.55 \mu \mathrm{m}$ and an effective area $A_{\text {eff }}=41 \mu \mathrm{m}^{2}$. The corresponding parameter for Eqs. (1) and (2) are $\beta_{2}=-47 \mathrm{ps}^{2} / \mathrm{km}$, $\beta_{3}=0.1 \mathrm{ps}^{3} / \mathrm{km}, \gamma=3 \times 10^{-3} \mathrm{~W} \mathrm{~m}^{-1}$ and $\gamma / \omega=2.6 \times 10^{-18} \mathrm{~s} / \mathrm{W} \mathrm{m}$ [15]. The amplitude is defined as $\sqrt{P_{\text {in }}}\left(P_{\text {in }}\right.$ is the input peak power of the pulse) and the cascaded couplers are numerically solved by Eqs. (1) and (2) using the fourth order Runge-Kutta method. The critical power is $P_{C}=109 \mathrm{KW}$ and the peak power for the first order soliton propagation is $P_{0}=4.56 \mathrm{KW}$.

\section{Study of energy transmission in the Mach-Zehnder interferometer}

As the Mach-Zehnder interferometer of Fig. 2 has two nonlinear couplers (dual core PCFs), this section presents a transmission study under the influence of the following parameters: pulse power, coupling length and phase shift. The transmission is defined as a function of the input signal by

$T_{k}=\frac{\int_{-\infty}^{\infty}\left|S_{k}(Z, t)\right|^{2} d t}{\left.\int_{-\infty}^{\infty} \mid S_{1}(0, t)\right)\left.\right|^{2} d t}$,

where $S_{k}(Z, t)$ are the output signals $(k=3,4)$ when the signal $S_{1}(0, T)$ is inserted in the port $S_{1}$. The parameter $T_{k}$ is the relation between the input signal energy and output signal energy $\left(S_{3}\right.$ and $S_{4}$ )

Now we will show how the coupler length modifies the curves of transmission as a function of the input power pulse. For this, a simulation is carried out fixing the phase controls $\left(\Delta \Phi_{1}=0\right.$ and $\left.\Delta \Phi_{2}=\pi / 2\right)$ and varying the input power pulse, $4.56 \mathrm{KW}<P_{\text {in }}<863.8 \mathrm{KW}$. Three simulations are performed with different coupler lengths $(L=0.9 \mathrm{~cm}, L=1.1875 \mathrm{~cm}$ and $L=1.35 \mathrm{~cm}$ ). In Fig. 3(a) are shown the transmissions $T_{3}$ and $T_{4}$ when $L=0.9 \mathrm{~cm}$. We note at $P_{\text {in }}=4.56 \mathrm{KW}$ that $T_{3}=0.5173$ and $T_{4}=0.4827\left(T_{3}+T_{4}=1\right.$, i.e., energy conservation). When the input power increases there is more energy in the A guide. $T_{3}=0.841$ is the maximum value at $721.9 \mathrm{KW}$. In Fig. 3(b), the curves $T_{3}$ and $T_{4}$ are illustrated to $L=1.1875 \mathrm{~cm}$. The behavior of the transmission in this simulation is different from the behavior shown in Fig. 3(a). Note that for $P_{i n}=4.56 \mathrm{KW}$ we have $T_{4}=0.42$ and $T_{3}=0.58$. The transmission $T_{3}$ is growing with input power until maximum value $T_{3}=0.8191$ in $656.5 \mathrm{KW}$. Then it decreases to $T_{3}=0.7801$ when 

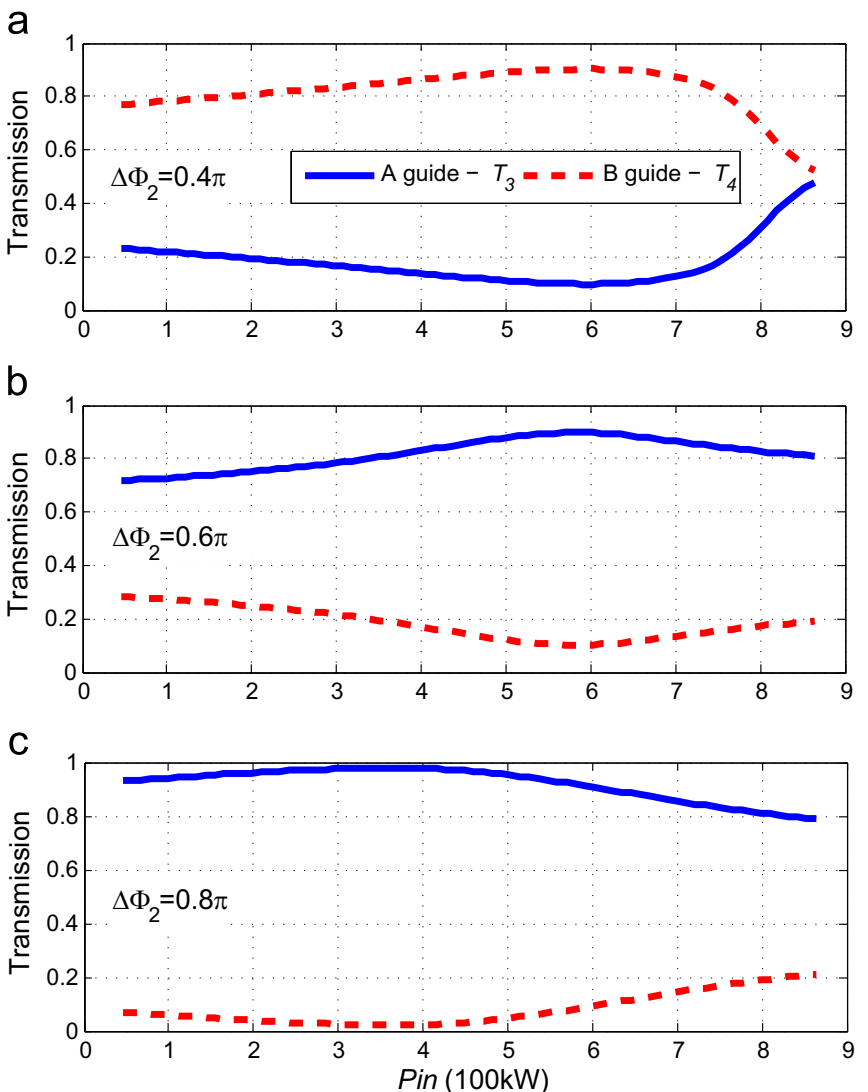

Fig. 4. The transmissions $T_{3}$ and $T_{4}$ as a function of the input power pulse $\left(P_{i n}\right)$. Three numerical simulations are realized for $\Delta \Phi_{2}=0.4 \pi$ (a), $\Delta \Phi_{2}=0.6 \pi$ (b) and $\Delta \Phi_{2}=0.8 \pi$ (c). The parameters $\Delta \Phi_{1}=0$ and $L=1.1875 \mathrm{~cm}$ are fixed for all simulations.

$P_{\text {in }}=863.8 \mathrm{KW}$. Finally, the transmissions $T_{3}$ and $T_{4}$ are shown in Fig. 3(c) when $L=1.35 \mathrm{~cm}$ in the simulation. We also note that the behavior of transmission is different from Fig. 3(b) and (c). When $P_{\text {in }}=4.56 \mathrm{KW}$, then $T_{3}=0.7423$ and $T_{4}=0.2577$. For higher input power values, we noticed a decrease of energy in the A guide. $T_{3}=0.5669$ is the minimum value when $P_{i n}=547.4 \mathrm{KW}$. After this, $T_{3}$ has a rapid growth reaching 0.8411 in $P_{\text {in }}=863.8 \mathrm{KW}$.

Fig. 4 shows the study of transmission varying the input power pulse to the three phase shifts after the first coupler: $\Delta \Phi_{2}=0.4 \pi$, $\Delta \Phi_{2}=0.6 \pi$ and $\Delta \Phi_{2}=0.8 \pi$. The coupler lengths are fixed at $L=1.1875 \mathrm{~cm}$ and the first phase shift is fixed too $\left(\Delta \Phi_{1}=0\right)$. In this study transmission the input power varies between $4.56 \mathrm{KW}$ and $863.8 \mathrm{KW}$. In Fig. 4(a) are shown the transmissions $T_{3}$ and $T_{4}$ when $\Delta \Phi_{2}=0.4 \pi$. We note that there is more energy in the B guide when $P_{\text {in }}=4.56 \mathrm{KW}\left(T_{4}=0.7724\right)$ and $T_{4}$ increases when $P_{i n}$ is increased until $623.8 \mathrm{KW}$. After this value, $T_{4}$ decreases reaching 0.5246 when $P_{\text {in }}=863.8 \mathrm{KW}$. However, when $\Delta \Phi_{2}=0.6 \pi$ (see Fig. 4(b)), we note more energy in the A guide to $P_{\text {in }}=4.56 \mathrm{KW}$. The energy is larger in this guide until $P_{\text {in }}=863.8 \mathrm{~W}$. In Fig. 4 (c) also the energy remains in the A guide. However, we note that for $P_{\text {in }}=362 \mathrm{KW}$ we have $T_{3}=0.9784$, i.e., almost all power remained in the A guide.

Fig. 5 shows the difference between the output pulse amplitudes and reference amplitude pulses in the ports $S_{3}$ and $S_{4}$. The "Reference line" divides the figure at bit 1 (up) and bit 0 (down). So, considering $i=3$ and 4 , when the amplitude of the output signal $\left(A_{S i}\right)$ is greater than the reference amplitude $\left(A_{r}\right)$ we obtain the bit 1, i.e., $\varepsilon_{\text {out }}=\left(A_{S i}-A_{r}\right)>0$ represents bit 1 . Then, likewise $\varepsilon_{\text {out }}=\left(A_{S i}-A_{r}\right)<0$ represents bit 0 . We analyzed the behavior of the output amplitude of the MZI according to the encoding parameter PAM-ASK. The encoding parameter variation analyzed was $0 \leq|\varepsilon| \leq 15 W^{1 / 2}$. From these results we can verify that the value of the coding parameter is not unique. Thus, it is possible to perform logic expressions, as well as fundamental all-optical logic gates from a MZI constituted of photonic crystal fiber. In order to justify this argument, we can see that the input $(0,1)$ and $(1,0)$ are always in different regions separated by the reference line in all the range of $\varepsilon$. However, we can surpass this point by inserting the phase control in the input pulses (as shown in Fig. 2). Our objective is to explore the MZI-PCF behavior around the reference line, because
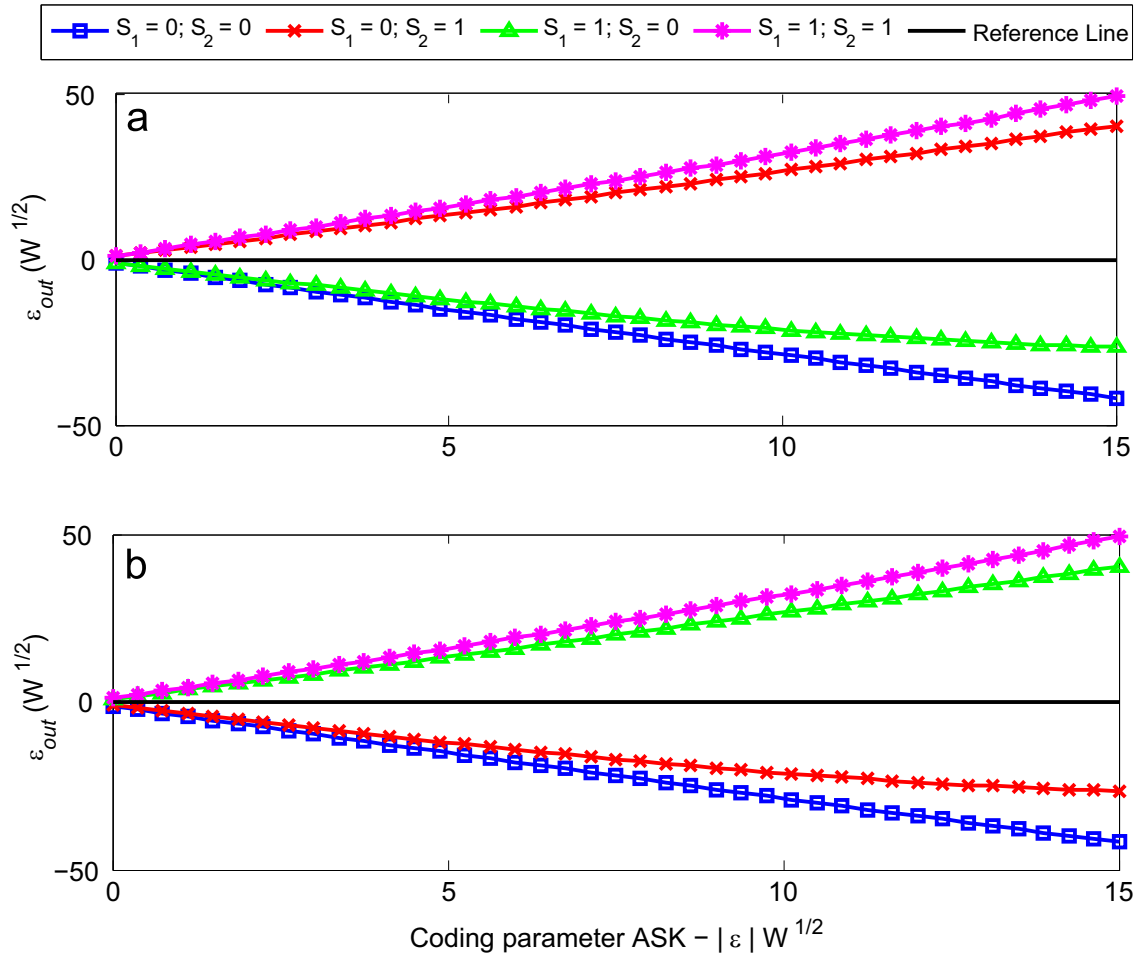

Fig. 5. Amplitude modulation measured on the output pulse $S_{3}$ (a) and $S_{4}$ (b) as a function of the coding parameter without phase control. 
the changes of the logic levels are most likely.

We conclude that the switching of the output pulses in the Mach-Zehnder interferometer is sensitive to adjustments in the coupler lengths, the phase shifts and the powers of the input pulses.

\section{Results and discussions}

The pulses inserted at $S_{1}$ and $S_{2}$ inputs pass through the modulator device where they are modulated to bit 0 or 1 , according to Eqs. (7) and (8). Then, they undergo its first phase shifting and pass through the first NLDC-PCF. Lastly, they undergo the second phase shifting, pass through the second NLDC-PCF and follow to the output ports. All logic gates were found investigating the output signals. In the following figures, analogous to Fig. 5, the "Reference line" divides the figure at bit 1 (up) and bit 0 (down). Then, considering $i=3$ and 4 , when the amplitude of the output signal $\left(A_{S i}\right)$ is greater than the reference amplitude $\left(A_{r}\right)$ we obtain the bit 1, i.e., $\varepsilon_{\text {out }}=\left(A_{S i}-A_{r}\right)>0$ represents bit 1 . Likewise $\varepsilon_{\text {out }}=\left(A_{S i}-A_{r}\right)<0$ represents bit 0 .

In Fig. 6 the coding parameter has been considered $|\varepsilon|=6 W^{1 / 2}$, the control phase $\Delta \Phi_{1}$ is fixed in $0.642 \pi$ and both couplers have length $L=1.1875 \mathrm{~cm}$. The phase shifting $\Delta \Phi_{2}$ is varying in the interval $[0,2 \pi]$. The $\varepsilon_{\text {out }}$ axis is defined as the difference between the amplitude of the output signal in $S_{4}, A_{S_{4}}$, and the amplitude of the reference pulse $A_{r}, \varepsilon_{o u t}=\left(A_{S_{4}}-A_{r}\right)$. The horizontal line in 0 named "Reference line" represents the bit transition. When $\varepsilon_{\text {out }}>0$ the pulse represents the bit 1 and when $\varepsilon_{\text {out }}<0$ it represents bit 0 . The analysis of these output signals are performed for each combination set of four inputs $\left[\left(S_{1}, S_{2}\right)=(0,0)\right.$, $\left.\left(S_{1}, S_{2}\right)=(0,1), \quad\left(S_{1}, S_{2}\right)=(1,0), \quad\left(S_{1}, S_{2}\right)=(1,1)\right]$. In the interval $[0.362 \pi, 0.693 \pi]$ (limited between the vertical dashed lines) note that only the curve representing $\left(S_{1}, S_{2}\right)=(1,1)$ is greater than zero. This mean that we have an AND gate in this interval. Only the input $(1,1)$ has output 1 , while the other inputs have output 0 .

In Fig. 7 we observed the $\varepsilon_{\text {out }}$ in port $S_{3}$ of the same experiment (coding parameter $|\varepsilon|=6 W^{1 / 2}$, the control phase $\Delta \Phi_{1}=0.642 \pi$ and $L=1.1875 \mathrm{~cm}$ for both couplers). Note that only the curve representing $\left(S_{1}, S_{2}\right)=(0,0)$ is smaller than zero in the interval $[0.306 \pi, 0.491 \pi]$ (between the dashed lines). This means that there is an OR gate in this interval in the port $S_{3}$. The phase $\Delta \Phi_{2}=0.4 \pi$ (marked by the vertical dot-dash line in Figs. 6 and 7) is contained in the intervals $[0.362 \pi, 0.693 \pi]$ and $[0.306 \pi, 0.491 \pi]$. Therefore, this MZI has two logic gates, AND and OR. To observe the pulses in the ports $S_{3}$ and $S_{4}$ we plot in Figs. 8 and 9 all output pulses when the inputs $\left[\left(S_{1}, S_{2}\right)=(0,0), \quad\left(S_{1}, S_{2}\right)=(0,1), \quad\left(S_{1}, S_{2}\right)=(1,0)\right.$, $\left.\left(S_{1}, S_{2}\right)=(1,1)\right]$ are modulated in the input.

In Figs. 8 and 9 we note high contrast in the pulse intensities. We also noticed that the shapes of the pulses have not changed significantly, because the power in the pulses is much lower than the critical power. When the carrier power is close to the critical power or the coding parameter is very high, then the amplitude begins to generate small peaks due to Raman scattering. Therefore, it was important for this result to avoid very high powers. The data these amplitudes can be found in Table 1.

Table 1 summarized the extracted data from Figs. 8 and 9. In the port $S_{3}$ the amplitude modulation parameter in the output, $\varepsilon_{\text {out }}$, has values equal to $-2.267 W^{1 / 2}$ (logic level 0 ), $4.879 W^{1 / 2}$, $5.593 W^{1 / 2}$ and $3.121 W^{1 / 2}$ (logic levels 1 ) for the distinct combinations of the input levels $\left(S_{1}, S_{2}\right)=(0,0), \quad\left(S_{1}, S_{2}\right)=(0,1)$, $\left(S_{1}, S_{2}\right)=(1,0),\left(S_{1}, S_{2}\right)=(1,1)$, respectively. These values confirm a logic gate OR. Similarly, in the port $S_{4}$ the amplitude modulation parameter, $\varepsilon_{\text {out }}$, has values equal to $4.233 W^{1 / 2}$ (logic level 1 ), $-8.695 W^{1 / 2},-1.919 W^{1 / 2}$ and $-2.136 W^{1 / 2}$ (logic levels 0 ), corresponding to a logic gate AND. In Table 1, we also report the contrast ratio $C R$ of these logic operations which gives the relative energy contained in the pulses corresponding to bits 1 and 0 , measured in decibels. For the OR operation, each pulse representing bit 1 is compared with the single pulse of bit 0 using the formula $10 \log _{10}\left(P / P_{\text {ZERO })}\right)$. Where the $P_{\text {(ZERO })}$ is the power of the output pulse in $S_{3}$ when the logic values $(0,0)$ are modulated in the input and $P$ is the power of the other output pulses. In this case, all results are positive because the bit 0 has lower energy. Similarly, each pulse representing bit 0 of the AND operation is compared with the pulse corresponding to bit 1 using the formula $10 \log _{10}\left(P / P_{(O N E)}\right)$. Where the $P_{(O N E)}$ is the power of the output pulse in $S_{4}$ when the logic values $(1,1)$ are modulated in the input and $P$

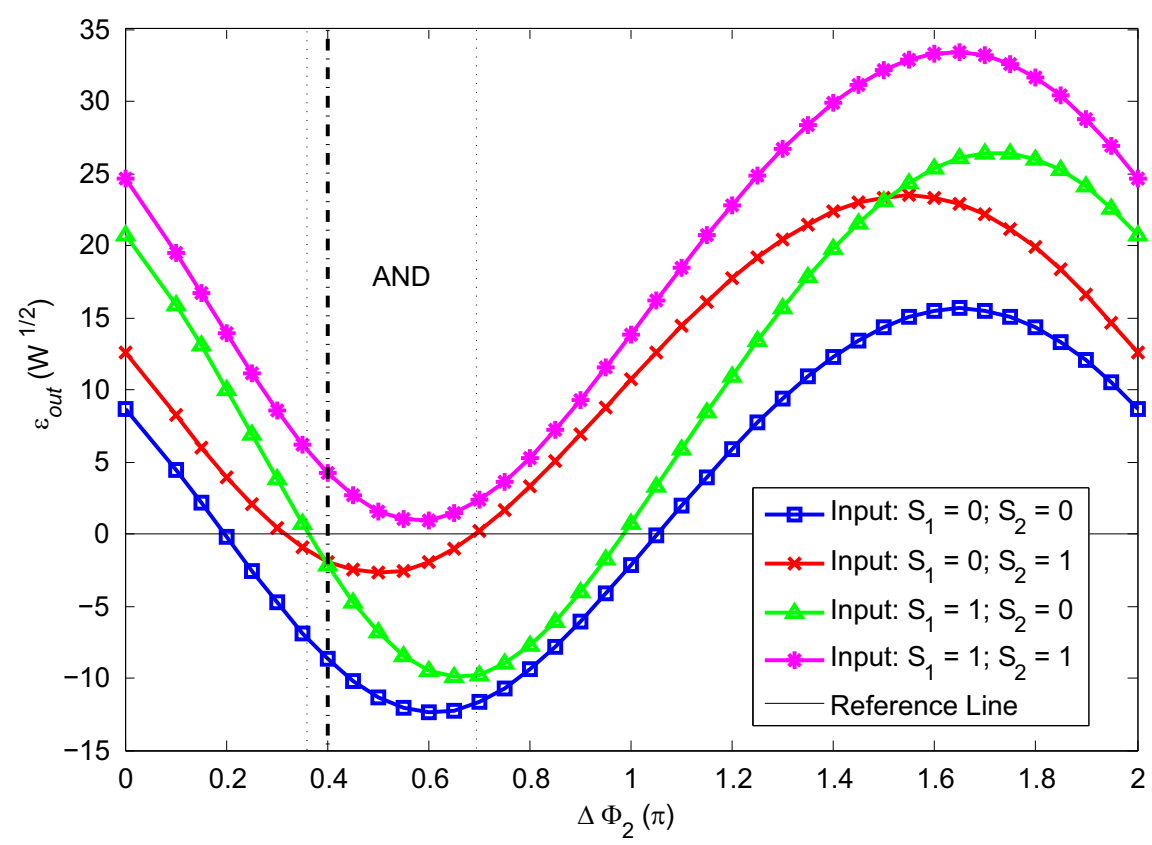

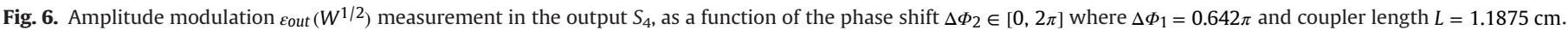
The AND gate is observed in the interval $[0.362 \pi, 0.693 \pi]$. The AND gate obtained when $\Delta \Phi_{2}=0.4 \pi$ is highlighted. 


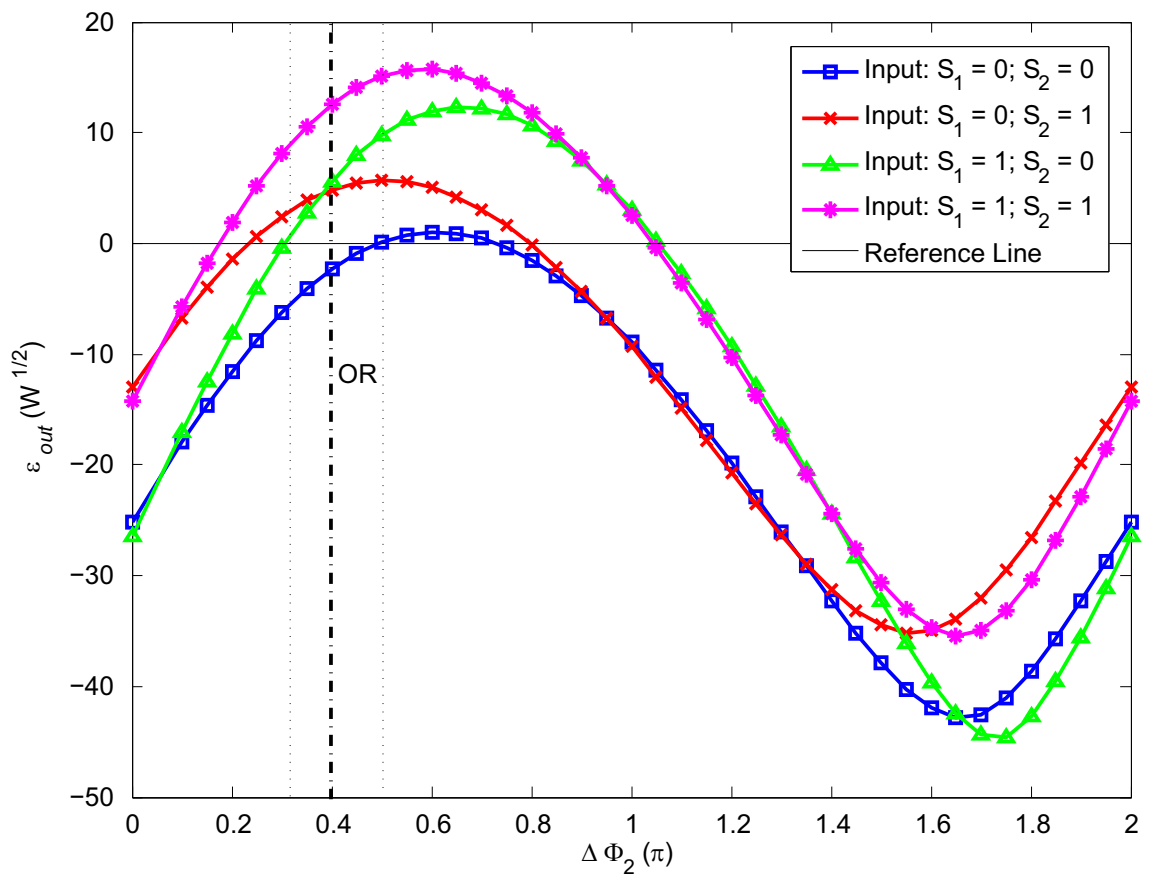

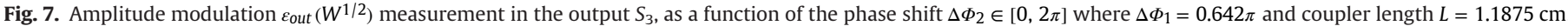
The OR gate is observed in the interval $[0.306 \pi, 0.491 \pi]$. The OR gate obtained when $\Delta \Phi_{2}=0.4 \pi$ is highlighted.

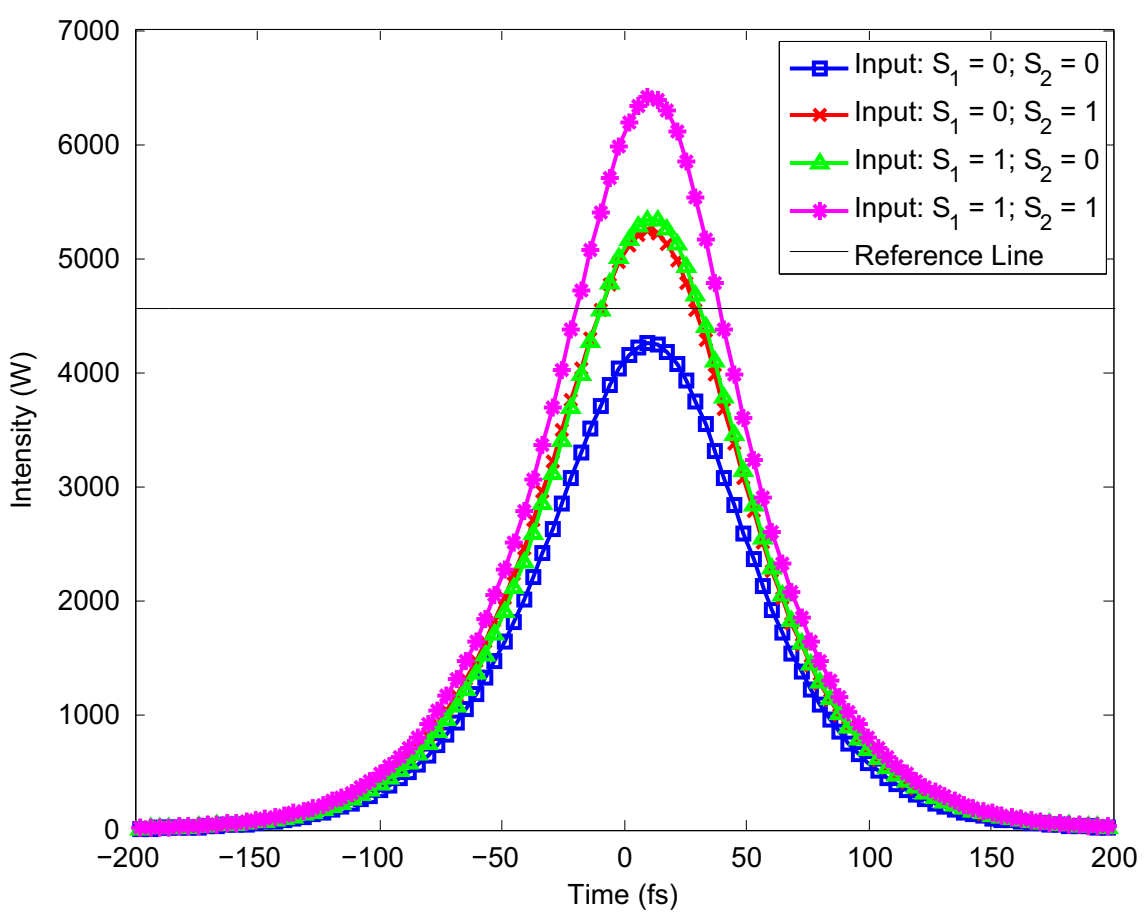

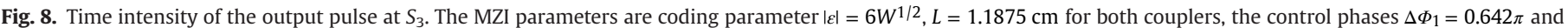
$\Delta \Phi_{2}=0.4 \pi$.

is the power of the other output pulses. In this case, all results are negative because the bit one has more energy. In the case of $C R$, the farther from zero, higher is the contrast.

\section{Conclusion}

This study is a numerical simulation of a Mach-Zehnder interferometer constituted of dual-core Photonic Crystal Fiber that behaves as a nonlinear directional coupler. The amplitude keying in the interferometer is studied to implement logic gates. Therefore, to represent the logic values 0 and 1, amplitude pulse modulation by amplitude shift-keying was used. The modulated pulses are the fundamental solitons of $100 \mathrm{fs}$. Nowadays, research in ultrashort pulses are necessary due to the crescent requirement of bandwidth in telecommunication systems.

On the whole, we conclude that ranging the power of the encoding parameter, the length of nonlinear photonic coupler and the phase modulators it is possible to control the amplitude keying in the Mach-Zehnder interferometer to obtain logic gates. 


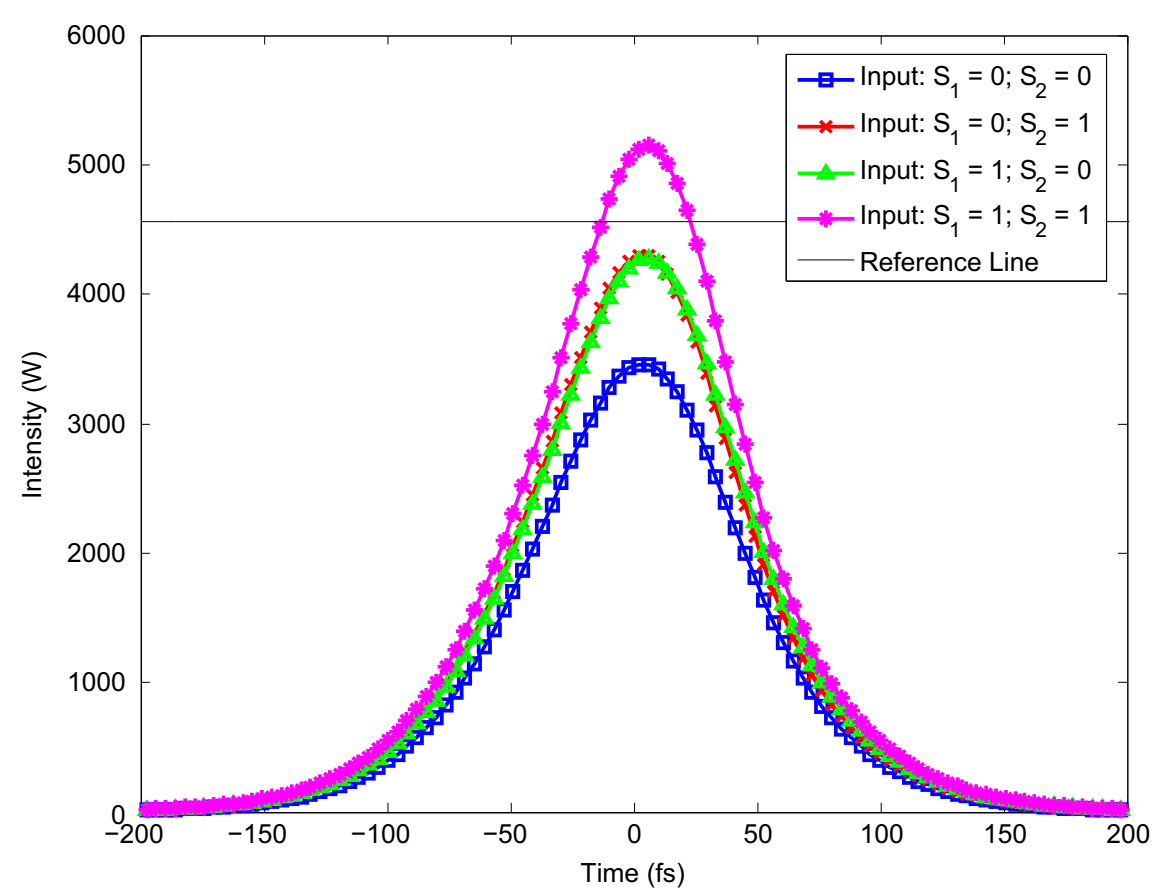

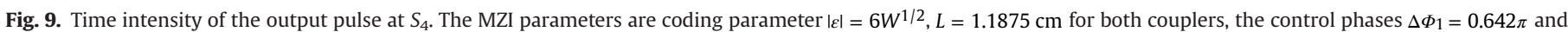
$\Delta \Phi_{2}=0.4 \pi$.

Table 1

Values corresponding the logic gates AND and OR. The AND and OR gates were obtained when $|\varepsilon|=6 W^{1 / 2}, L=1.1875 \mathrm{~cm}, \Delta \Phi_{1}=0.642 \pi$ and $\Delta \Phi_{2}=0.4 \pi$.

\begin{tabular}{lllll}
\hline$S_{1}$ & $S_{2}$ & $S_{4}$ & $\varepsilon_{\text {out }}\left(W^{1 / 2}\right)$ & CR $(\mathrm{dB})$ \\
\hline AND & & & & \\
0 & 0 & 0 & -8.695 & -2.34 \\
0 & 1 & 0 & -1.919 & -1.50 \\
1 & 0 & 0 & -2.136 & -1.49 \\
1 & 1 & 1 & 4.233 & \\
OR & & & & \\
0 & 0 & 0 & -2.267 & 0.85 \\
0 & 1 & 1 & 5.879 & 0.84 \\
1 & 0 & 1 & 12.544 & 1.56 \\
1 & 1 & 1 & & \\
\hline
\end{tabular}

Moreover, there exist two inputs and two outputs in the interferometer, we demonstrate that it is possible to find two logic gates in a single configuration of the Mach-Zehnder interferometer constituted of dual-core PCF. This result is not common and it is useful in saving space in complex circuit designs.

The AND and OR gates are found when we configure the MachZehnder interferometer with the following values: the encoding parameter $|\varepsilon|=6 W^{1 / 2}$, the length of the couplers are $L=1.1875 \mathrm{~cm}$, the phase modulators $\Delta \Phi_{1}=0.642 \pi$ and $\Delta \Phi_{2}=0.4 \pi$. In this configuration, when we modulate all logic values of Truth Table, the contrast ratio is high in all output pulses. Hence, the two logic gates presented in this paper have high performance.

\section{Acknowledgment}

This work was supported by Brazilian Agencies FUNCAP and CAPES.

\section{References}

[1] P. Russell, Photonic crystal fibers, Science 299 (5605) (2003) 358-362.

[2] D. Skryabin, F. Luan, J. Knight, P.S.J. Russell, Soliton self-frequency shift cancellation in photonic crystal fibers, Science 301 (5640) (2003) 1705-1708.

[3] J. Herrmann, D. Griebner, N. Zhavoronkov, A. Husakou, D. Nickel, G. Korn, J. Knight, W. Wadsworth, P. Russel, Experimental evidence for supercontinuum generation by fission of higher-order solitons in photonic crystal fibers, in: Summaries of Papers Presented at the Quantum Electronics and Laser Science Conference, 2002. QELS'02. Technical Digest, IEEE, Long Beach, CA, 2002, pp. 165-166.

[4] J. Menezes, W. de Fraga, G. Guimarães, A. Ferreira, H. Rocha, M. Da Silva, A. Sombra, Optical switches and all-fiber logical devices based on triangular and planar threecore nonlinear optical fiber couplers, Opt. Commun. 276 (1) (2007) 107-115.

[5] B. Mukherjee, Wdm optical communication networks: progress and challenges, IEEE J. Sel. Areas Commun. 18 (10) (2000) 1810-1824.

[6] W. Fraga, J. Menezes, M. Da Silva, C. Sobrinho, A. Sombra, All optical logic gates based on an asymmetric nonlinear directional coupler, Opt. Commun. 262 (1) (2006) 32-37.

[7] Q. Lai, M. Lanker, W. Hunziker, H. Melchior, Tunable wavelength selection switch and multiplexer/demultiplexer based on asymmetric silica-on-silicon Mach-Zehnder interferometer, Electron. Lett. 34 (3) (1998) 266-267.

[8] K.R. Khan, S. Bidnyk, T.J. Hall, Tunable all optical switch implemented in a liquid crystal filled dual-core photonic crystal fiber, Prog. Electromagn. Res. M 22 (2012) 179-189.

[9] M. Liu, K.S. Chiang, Propagation of ultrashort pulses in a nonlinear two-core photonic crystal fiber, Appl. Phys. B 98 (4) (2010) 815-820.

[10] M.B.C. Costa, A.M. Bastos, A.G. Coelho, C.S. Sobrinho, M.L. Lyra, A.S.B. Sombra, High contrast optical "OR" logic gates using a photonic crystal fiber modulated by PAMASK, J. Opt. Commun. 35 (2014) 85-94.

[11] A.d.C. Ferreira, C. Sobrinho, J. Menezes, W. Fraga, H. Rocha, A. Wirth, L.K. Sabóia, G. Guimarães, J. Filho, A. Sombra, A performance study of an all-optical logic gate based in pam-ask, J. Mod. Opt. 56 (8) (2009) 1004-1013.

[12] A. Coelho Jr., M. Costa, A. Ferreira, M. da Silva, M. Lyra, A. Sombra, Realization of alloptical logic gates in a triangular triple-core photonic crystal fiber, J. Lightwave Technol. 31 (5) (2013) 731-739.

[13] J. Sousa, A. Ferreira, G. Batista, C. Sobrinho, A. Bastos, M. Lyra, A. Sombra, et al. Generation of logic gates based on a photonic crystal fiber Michelson interferometer, Opt. Commun. 322 (2014) 143-149.

[14] B. Kim, T.-H. Kim, L. Cui, Y. Chung, Twin core photonic crystal fiber for in-line MachZehnder interferometric sensing applications, Opt. Express 17 (18) (2009) 15502-15507.

[15] W. Shin, Y.L. Lee, B.-A. Yu, Y.-C. Noh, T.J. Ahn, Highly sensitive strain and bending sensor based on in-line fiber Mach-Zehnder interferometer in solid core large mode area photonic crystal fiber, Opt. Commun. 283 (10) (2010) 2097-2101.

[16] K.S. Chiang, Propagation of short optical pulses in directional couplers with Kerr nonlinearity, J. Opt. Soc. Am. B 14 (6) (1997) 1437-1443.

[17] G. Agrawal, Applications of Nonlinear Fiber Optics, Academic Press, Burlington, MA, 2001.

[18] S. Droulias, M. Manousakis, K. Hizanidis, Switching dynamics in nonlinear directional fiber couplers with intermodal dispersion, Opt. Commun. 240 (1) (2004) 209-219.

[19] A. Kumar, A.K. Sarma, Femtosecond-soliton switching in a three-core coupler, Jpn. J. Appl. Phys. 44 (12R) (2005) 8498. 